\title{
Role of circular RNAs in osteoarthritis (Review)
}

\author{
JICHENG WANG, BO YANG, CHANGKUN WU, YONGZHI GUO, \\ XIN JIANG and YANGYANG ZHANG
}

Department of Joint Surgery, Weifang People's Hospital, Weifang, Shandong 261000, P.R. China

Received April 12, 2021; Accepted August 11, 2021

DOI: $10.3892 /$ etm.2021.10714

\begin{abstract}
Osteoarthritis (OA) is a chronic bone and joint disease characterized by articular cartilage degeneration and joint inflammation. OA is the most common form of arthritis, and the major clinical manifestations of OA are chronic pain and joint activity disorder, which severely affect patients' quality of life. Circular RNA (circRNA) is a type of non-coding RNA that is ubiquitous in eukaryotic cells. Unlike standard linear RNAs, they form a covalently closed continuous loop without 5' or 3' polarity. They are usually considered as byproducts of mis-splicing or mRNA processing. CircRNAs have been detected and identified in numerous species. Various studies have confirmed that certain circRNAs are differentially expressed in OA cartilage and are closely associated with a variety of pathological processes of OA, including extracellular matrix degradation, inflammation and apoptosis. The present study reviewed the latest research on circRNAs in the pathogenesis of OA, providing a novel direction for the prevention, diagnosis and treatment of OA.
\end{abstract}

\section{Contents}

1. Introduction

2. Generation and biological functions of circRNAs

3. Role of circRNAs in OA

4. Promotion of OA pathogenesis

5. Inhibition of OA pathogenesis

6. Conclusions and perspectives

Correspondence to: Professor Yangyang Zhang, Department of Joint Surgery, Weifang People's Hospital, 151 Guangwen Street, Kuiwen, Weifang, Shandong 261000, P.R. China

E-mail: zhangyy20212021@163.com

Abbreviations: ceRNA, competing endogenous RNA; ECM, extracellular matrix; circRNA, circular RNA; miRNA, microRNA; OA, osteoarthritis; RBP, RNA binding protein; Col II, type II, collagen

Key words: osteoarthritis, circular RNA, pathogenesis, biomarkers, diagnosis, treatment

\section{Introduction}

Osteoarthritis (OA) is a chronic bone and joint disease characterized by articular cartilage degeneration and joint inflammation (1). OA is the commonest form of arthritis, and the major clinical manifestations of OA are chronic pain and joint activity disorder (1), which severely affect patients' quality of life. OA is most common in middle-aged and elderly subjects $(2,3)$. The pathogenesis of OA is complex, and its etiology remains to be fully elucidated. At present, the occurrence of OA is assumed to be associated with various risk factors, including mechanical, genetic and physical factors (4-6). A number of these factors, including age, sex, obesity and bone density, increase the risk of OA, and age has been reportedly indicated to be an independent risk factor (4-6). Current treatments for OA aim to alleviate or control pain, delay or prevent disease progression, improve or reconstruct joint function, correct deformity and maintain patients' quality of life (7). OA treatment is based on the combination of the disease education, sports activity guidance, medication and, if necessary, surgical intervention. In addition, an individualized treatment plan may be developed considering the patient's age, sex, OA location, extent, symptoms and underlying comorbidities (7). However, the clinical results obtained by conventional treatments are poor, and the risk of side effects is high, including postoperative pain, fractures and dislocations (7). Therefore, elucidating the pathological mechanism of OA may be helpful in identifying novel specific biomarkers that may contribute to the development of effective treatments for managing OA symptoms.

Circular RNA (circRNA) is a type of non-coding RNA that is ubiquitous in eukaryotic cells. Unlike standard linear RNAs, circRNAs form a covalently closed continuous loop without $5^{\prime}$ or 3' polarity (8). They are usually considered as byproducts of mis-splicing or mRNA processing (9). In the present review, the latest research on circRNAs was summarized with respect to the pathogenesis of $\mathrm{OA}$, to provide a novel direction for the prevention, diagnosis and treatment of OA.

\section{Generation and biological functions of circRNAs}

Sanger et al (10) and Hsu and Coca-Prados (11), using electron microscopy, were the first to discover circRNAs in plant-based and eukaryotic organisms in the 1970s. Subsequently, circRNA [in Hepatitis $\delta$ virus (HDV)] was amplified by PCR and sequenced to confirm that it is also expressed in humans (12). 
Several studies have confirmed that circRNAs are differentially expressed in OA cartilage and are closely related to the pathological processes of OA, including extracellular matrix (ECM) degradation, inflammation and apoptosis $(12,13)$. With the development of RNA sequencing and bioinformatics, thousands of circRNAs have been discovered in various organisms including plants, animals and viruses) (13) and each circRNA is considered to have a regulatory effect on a variety of biological processes.

According to the genomic origin and structural characteristics of circRNAs, they are divided into three types: Exon circRNA, exon-intron circRNA and intron circRNA $(14,15)$. CircRNAs are produced through a highly complex process that involves different cyclization mechanisms.

Usually, the spliceosome operates for eukaryotic pre-messenger RNA (pre-mRNA), which removes introns and junction exons to produce linear RNA transcripts with $5^{\prime}$ or 3' polarity (16). Most circRNAs are produced through the reverse splicing process, which constitutes the difference between circRNA and linear RNA standard splicing; the resultant sequence does not follow the $5^{\prime}-3^{\prime}$ order $(16,17)$. In the same pre-mRNA, the exons located between the downstream 5'-splice site (splice donor) and the upstream 3'-splice site (splice acceptor) are produced without end structures (such as 5' cap structure or poly A tail) to yield the ring product $(14,18,19)$. Two different exon cyclization mechanism models were proposed by Jeck et al (14) in 2013. The first model is the lasso-driven circularization or exon skipping. The characteristic of this model is that the original non-adjacent exons come nearer by partially folded pre-mRNA transcripts and are close to other exons, leading to exon skipping and formation of overlapping regions, thereby creating an area containing exons and an internal lasso intermediate. The introns in the lasso are removed, resulting in exon circRNA $(14,20)$. Usually, the introns located between the circularized exons are spliced, and in certain cases the exon-intron circRNA is not spliced (20). The second model refers to cyclization driven by intron pairing or direct reverse splicing. The model involves complementarity that spans flanking introns or other RNA secondary structure base assignments and connects downstream splice donors with upstream splice acceptors to form a loop structure (20). The intron circRNA produced by the intron lasso is able to resist the degradation of debranching enzymes $(14,20)$. The intron circRNA contains a unique $3^{\prime}-5$ ' connection, while the exon circRNA lacks this unique connection. The 5'-splice site that is rich in 7 nucleotides $\mathrm{GU}$ and the sequence close to the $\mathrm{C}$-rich branch of 11 nucleotides form the junction site (Fig. 1) (21).

Studies confirmed the circRNA biogenesis model through RNA binding proteins (RBPs) $(22,23)$. In this model, two flanking introns combine with quaking protein and muscleblind-like protein 1 to form two flanking intron sequences that form a bridging point and promote cyclization to produce circRNA $(22,23)$. This mechanism is similar to the circularization pathway driven by intron pairing, except that RBP regulates adjacent splice sites rather than the direct base pairing between complementary motifs seen in the intron pairing-driven model.

Several studies have confirmed that circRNAs may act as microRNA (miRNA/miR) sponges, interact with RBP and regulate gene transcription, and certain circRNAs may be translated into proteins or peptides $(14,20,21,24-29)$. Therefore, as illustrated in Fig. 2, the major functions of circRNAs may be summarized as follows: i) miRNA sponge-as a type of linear non-coding RNA, miRNAs may silence or degrade target mRNAs through base pairing, thereby regulating the pathological and physiological processes of organisms (30). CircRNAs may be used as competing endogenous RNAs (ceRNAs) that bind to miRNAs, inhibits the binding of miRNAs to the target and thereby inhibits mRNA translation $(31,32)$; ii) combination with RBPs-circRNA binds to RBP to form an RNA-protein complex, which regulates RBP and interacts with linear RNA to perform biological functions $(31,32)$; iii) regulation of gene transcription-numerous circRNAs are present in the nucleus, which are able to bind to RBPs, particularly with transcription-related factors, such as RNA polymerase II, and transcription factors, and recruit transcription-related factors into the parental genes, thereby affecting the expression of the parental genes and regulating the transcription process (33); and iv) translation into protein or peptide-evidence suggests that certain circRNAs may be also translated into proteins. When the synthetic circular RNA contains an efficiently translated internal ribosome entry site sequence, the circular RNA directly binds to the ribosome and is translated in eukaryotic cells $(22,34)$. Another study confirmed that methylated adenosine N6 may drive natural eukaryotic endogenous circRNA protein translation, which indicates that certain circRNAs exhibit a protein-encoding function $(35,36)$.

\section{Role of circRNAs in OA}

CircRNAs have been reportedly involved in the development and process of several human diseases, such as diabetes, cardiovascular disease, cancer and Alzheimer's disease $(37,38)$. However, the role of circRNAs in the pathogenesis of OA remains to be fully elucidated. Previous studies have indicated that certain circRNAs are abnormally expressed in human OA articular cartilage compared with cartilage tissue of healthy controls.

Li et al (39) used whole-transcriptome sequencing and determined that 42 types of circRNAs may be differentially expressed in OA cartilage tissue. Another recent study identified 122 circRNAs that were differentially expressed in OA through RNA sequencing; five downregulated circRNAs and an upregulated circRNA were confirmed by reverse transcription-quantitative (RT-q)PCR (40). Xiao et al (41) used the Illumina sequencing platform to detect the expression of circRNA in the OA knee joint and identified a total of 197 differentially expressed circRNAs, including hg38_circ_0007474 and hg38_circ_0000118, and RT-qPCR confirmed that three circRNAs were related to OA (hsa_circ_0002485, hsa circ_0005567 and hsa_circ_0045714). Another previous study suggested that 71 circRNAs were differentially expressed in OA cartilage (42). Furthermore, 16 and 55 of these circRNAs were upregulated and downregulated in OA cartilage tissues, respectively. These differentially expressed circRNAs were indicated to be involved in the pathological process of OA in cartilage tissues (42). These studies suggested that abnormal expression of circRNAs in human OA cartilage is involved in the pathogenesis of OA. These differentially expressed 


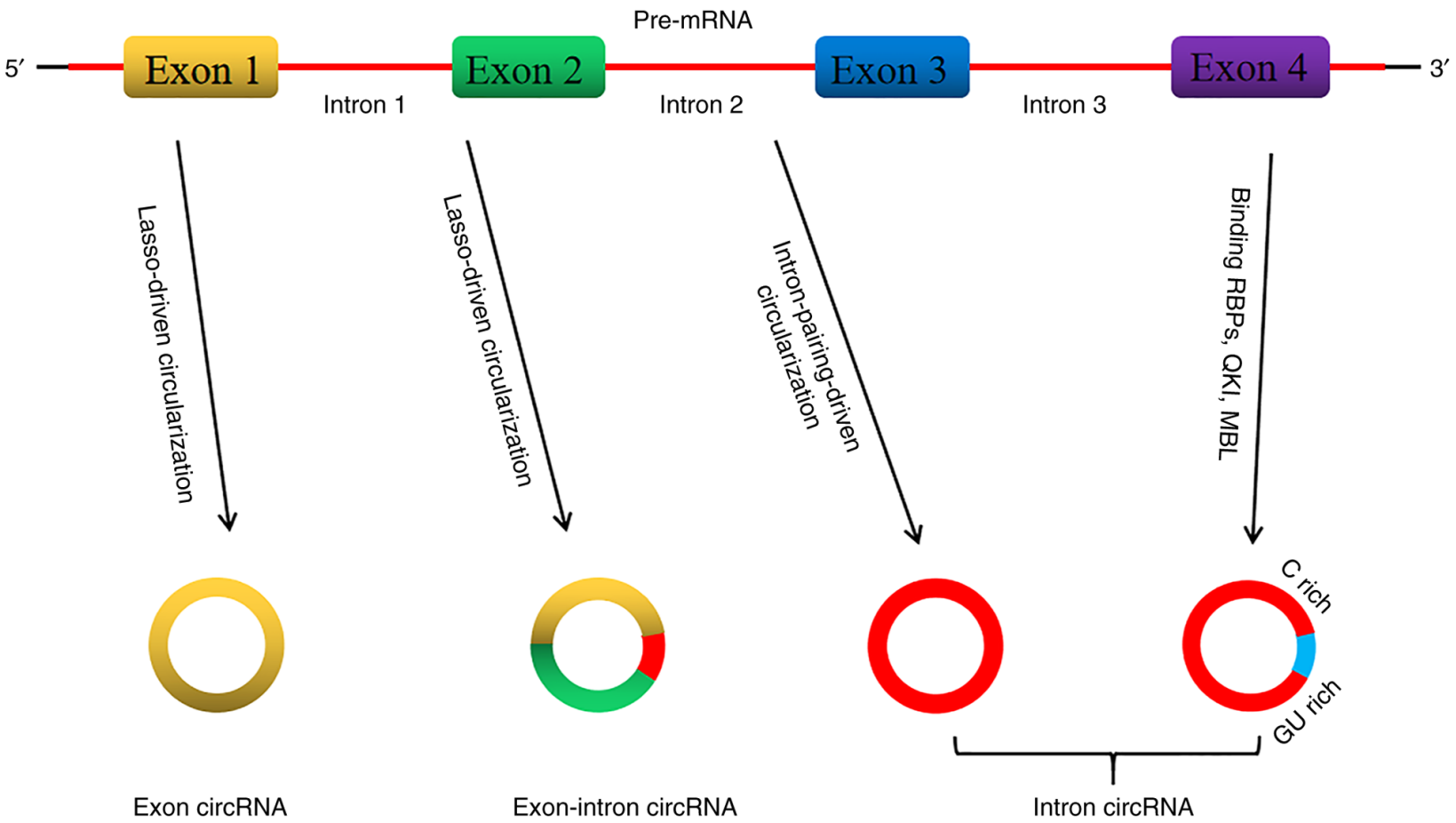

Figure 1. Biogenesis models of circRNAs. According to the genomic origin and structural characteristics of circRNAs, they are divided into three types: Exon circRNA, exon-intron circRNA and intron circRNA. The first model is the lasso-driven circularization or exon skipping. The second model refers to cyclization driven by intron pairing or direct reverse splicing. The third model refers to the circRNA biogenesis model through RNA binding proteins (RBPs). In this model, two flanking introns combine with quaking protein and muscleblind-like protein 1 to form two flanking intron sequences that form a bridging point and promote cyclization to produce circRNA. This mechanism is similar to the circularization pathway driven by intron pairing, except that RBP regulates adjacent splice sites rather than the direct base pairing between complementary motifs seen in the intron pairing-driven model. RBP, RNA-binding protein; circRNA, circular RNA; QKI, quaking protein; MBL, muscleblind protein.

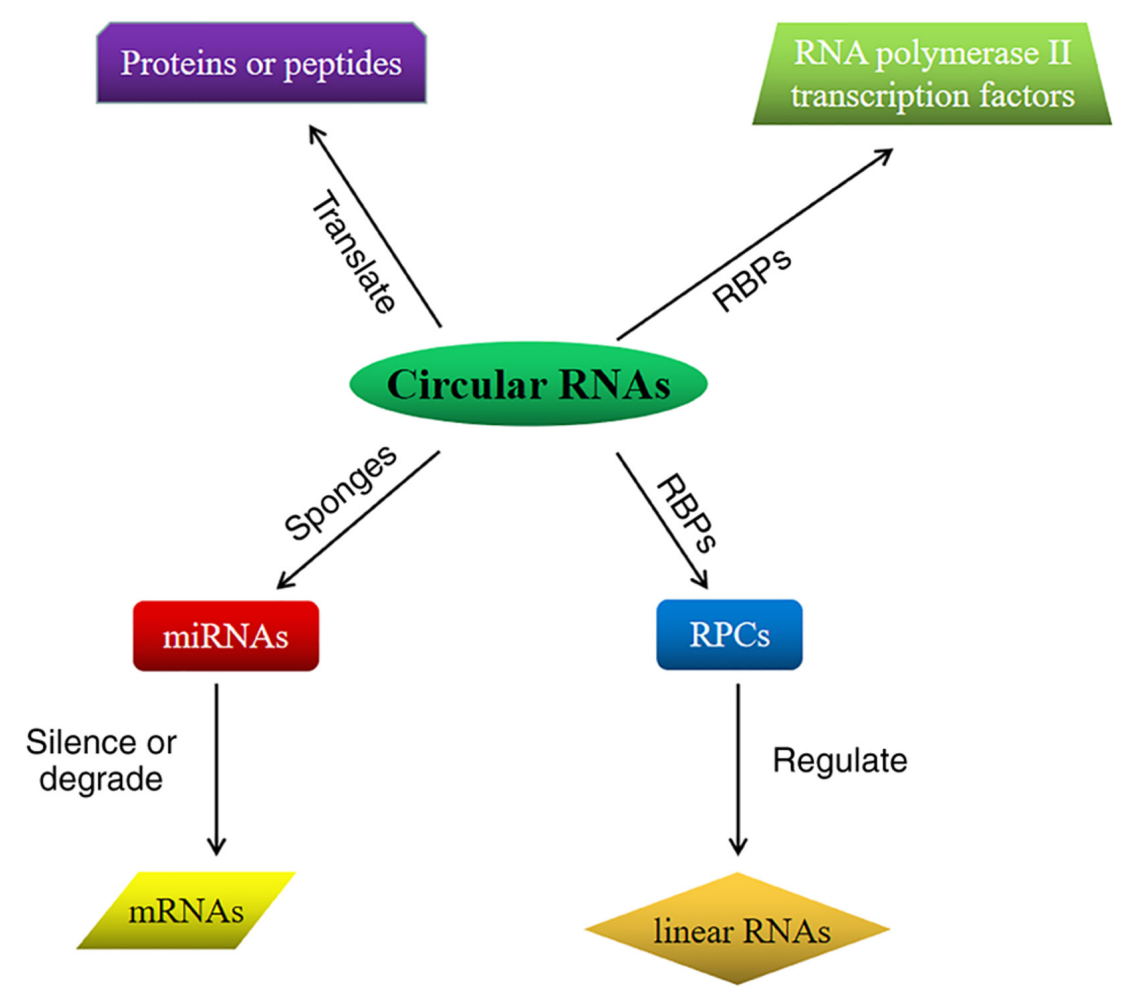

Figure 2. Biological function of circular RNAs. i) miRNA sponge-CircRNAs may be used as competing endogenous RNAs (ceRNAs) that bind to miRNAs, inhibit the binding of miRNAs to the target and thereby inhibits mRNA translation; ii) combination with RBPs-circRNA binds to RBP to form an RNA-protein complex, which regulates RBP and interacts with linear RNA to perform biological functions; iii) regulation of gene transcription-circRNAs bind to RBPs, recruit transcription-related factors into the parental genes, thereby affecting the expression of the parental genes and regulating the transcription process; and iv) translation into protein or peptide-certain circRNAs may be also translated into proteins. RBP, RNA-binding protein; RPCs, RNA-protein complexes; miRNA, microRNA. 
circRNAs can either promote or inhibit the occurrence and development of OA.

\section{Promotion of $\mathrm{OA}$ pathogenesis}

It has been indicated that the increased catabolism of ECM articular cartilage accelerates the development of OA (43). Type II collagen (Col II) and aggrecan are important components of the ECM. The major pathological feature of OA is the decrease of Col II and aggrecan. The main enzymes responsible for ECM degradation are disintegrin and metalloproteinase with thrombospondin-like motifs (ADAMTS) and matrix metalloproteinases (MMPs) (44). In addition, numerous MMPs are highly upregulated in OA cartilage and knocking down ADAMTS may reduce cartilage destruction (44). Further evidence has indicated that inflammation is another important driving factor of OA cartilage pathology $(45,46)$. The inflammatory process of $\mathrm{OA}$ involves traditional pro-inflammatory mediators, including IL-1 $\beta$ and TNF- $\alpha$ and other chemokines, such as nicotinamide phosphoribosyltransferase (47). It has been indicated that the decrease in the number of chondrocytes induced by apoptosis is an important factor leading to the degeneration of OA cartilage (9).

As circRNAs have a wide range of biological functions, they may have key regulatory roles in the occurrence and development of OA by regulating cartilage matrix metabolism and inflammation, along with chondrocyte proliferation, differentiation and apoptosis (44-47).

CircRNA-CER is a special type of ECM-related chondrocyte circRNA (48). Through network analysis of circRNA-miRNA-mRNA interactions, five miRNAs with potential binding sites for circRNA-CER were identified: miR-636, miR-665, miR-217, miR-646 and miR-136. Further investigation indicated that after miR-136 was combined with circRNA 3'-UTR, the expression of circRNA-CER and the MMP13 gene was jointly regulated. Application of small interfering (si)RNA to knockdown circRNA-CER (si-circRNA-CER) in OA chondrocytes significantly reduced the mRNA expression of MMP13. Co-transfection with miR-136 inhibitor and si-circRNA-CER reduced MMP13 expression and increased the mRNA expression of Col II and proteoglycan (such as cartilage proteoglycan) compared with cartilage tissue of healthy controls (42). This indicated that circRNA-CER may be used as a ceRNA to regulate the expression of MMP13 and participate in the degradation of chondrocyte ECM (42). Therefore, inhibiting circRNA-CER is likely a novel treatment strategy for stimulating ECM regeneration and delaying joint degeneration.

CircRNA-MSR is another special type of cartilage-related circRNA. It has been indicated that circRNA-MSR is highly expressed in the damaged cartilage area in OA (49). At the same time, its expression also notably increases under the mechanical stress of chondrocytes. Therefore, it is also called circRNA related to mechanical stress (49). Following knockdown of circRNA-MSR, the expression of TNF- $\alpha$ was inhibited, while that of Col II and aggrecan was increased, indicating that circRNA-MSR is able to promote ECM degradation. Further studies have confirmed that the 3'-UTR of circRNA-MSR and TNF- $\alpha$ were complementary to miR-875, which suggested that the common target miRNA of circRNA-MSR and TNF- $\alpha$ is miR-875 (49). Knockdown of circRNA-MSR may inhibit ECM degradation and reduce inflammation.

Wu et al (50) confirmed that hsa_circ_0005105 contains the binding site of miR-26a. It was demonstrated that hsa_circ_0005105 was highly expressed in IL-1 $\beta$-induced OA chondrocytes, while the expression of miR-26a was downregulated, indicating that hsa_circ_0005105 likely participates in the biological process of OA through miR-26a (50). Hsa_ circ_0005105 has been indicated to inhibit the expression of Col II and aggrecan and promote the expression of MMP13 and ADAMTS4, whereas miR-26a exhibited the opposite effect (50). Hsa_circ_0005105 promoted ECM degradation by regulating the expression of miR-26a. Therefore, the study by Wu et al (50) suggested that hsa_circ_0005105 promoted the development and process of OA.

Zhou et al (51) discovered a circRNA from the mouse Atp9b locus, which was named circRNA_Atp9b (circ_15898). After stimulation with IL-1 $\beta$, the expression of circRNA_Atp9b in mouse chondrocytes was markedly increased. Knockout of circRNA_Atp9b promoted the expression of Col II while inhibiting the production of MMP13, cyclooxygenase-2 and IL-6. Their study also indicated that circRNA_Atp9b acts as miR-138-5p ceRNA in IL-1 $\beta$-induced chondrocytes (51). CircRNA_Atp9b directly targets miR-138-5p and knockdown of miR-138-5p reversed the effect of circRNA_Atp9b on ECM degradation and inflammation (51). In summary, these results indicated that circRNA_Atp9b, which functions as a sponge for miR-138-5p, promoted the progression of OA by regulating ECM catabolism and chondrocyte inflammation.

Another study suggested that in the cartilage tissue of an OA mouse model treated with IL-1 $\beta$, circRNA.33186 was markedly upregulated (52). After knocking down circRNA.33186, the expression of Col II increased and the expression of MMP-13 decreased. At the same time, knockdown of circRNA.33186 promoted the proliferation of chondrocytes treated with IL-1 $\beta$ and inhibited apoptosis (52). Further investigation demonstrated that circRNA.33186 could directly bind to and inhibit miR-127-5p, increase the expression of MMP-13 and promote OA (52). These results suggested that circRNA.33186 is a potential drug target for OA treatment.

Chen et al (53) reported that circRNA-UBE2G1 and hypoxia-inducible factor (HIF)-1 $\alpha$ were overexpressed in OA cartilage tissues, while the expression level of miR-373 was downregulated compared with healthy cartilage tissues. Further research indicated that circRNA-UBE2G1 acts as a ceRNA to bind to miR-373 and HIF- $1 \alpha$ is the target of miR-373. Thus, circRNA-UBE2G1 promotes OA progression by regulating the miR-373/HIF- $1 \alpha$ axis (53).

Another recent study suggested that circ_0136474 is involved in the pathological process of OA (54). RT-qPCR detection revealed that circ_0136474 and DNA methyltransferase 3A (DNMT3A) were both overexpressed in OA cartilage tissue, while the expression of miR-766-3p was reduced compared with healthy cartilage tissues (54). Further research indicated that DNMT3A is the target of miR-766-3p. Inhibiting the expression of circ_0136474 was able to reduce oxidative damage in OA chondrocytes by regulating the miR-766-3p/DNMT3A axis (54).

$\mathrm{Ni}$ et al (55) indicated that circPSM3 is highly expressed in OA chondrocytes. Further research suggested that circPSM3 
Table I. Roles of circRNAs in the promotion of the pathogenesis of osteoarthritis.

\begin{tabular}{|c|c|c|c|}
\hline CircRNA & Target & Biological function & Refs \\
\hline circRNA-CER & miR-136 & $\begin{array}{l}\text { Degradation of ECM, } \\
\text { promotion of inflammation }\end{array}$ & $(42)$ \\
\hline circRNA-MSR & $\operatorname{miR}-875$ & $\begin{array}{l}\text { Degradation of ECM, } \\
\text { promotion of inflammation }\end{array}$ & (49) \\
\hline hsa_circ_0005105 & miR-26a & Degradation of ECM & $(50)$ \\
\hline circRNA_Atp9b & $\operatorname{miR}-138-5 p$ & $\begin{array}{l}\text { Degradation of ECM, } \\
\text { promotion of inflammation }\end{array}$ & $(51)$ \\
\hline circRNA.33186 & $\operatorname{miR}-127-5 p$ & Degradation of ECM & $(52)$ \\
\hline circRNA-UBE2G1 & $\operatorname{miR}-373$ & $\begin{array}{l}\text { Degradation of ECM, } \\
\text { promotion of apoptosis }\end{array}$ & $(53)$ \\
\hline circ_0136474 & $\operatorname{miR}-766-3 p$ & $\begin{array}{l}\text { Degradation of ECM, } \\
\text { promotion of apoptosis }\end{array}$ & (54) \\
\hline circPSM3 & miR-296-5p & Inhibition of cell proliferation and differentiation & $(55)$ \\
\hline circRNA-CDR1as & miR-641 & $\begin{array}{l}\text { Degradation of ECM, } \\
\text { promotion of inflammation }\end{array}$ & $(56)$ \\
\hline circGCN1L1 & $\operatorname{miR}-330-3 p$ & $\begin{array}{l}\text { Reduction of ECM generation, promotion of } \\
\text { inflammation }\end{array}$ & $(57)$ \\
\hline
\end{tabular}

ECM, extracellular matrix; miR, microRNA; circ/circRNA, circular RNA.

inhibits the proliferation and differentiation of OA chondrocytes by targeting miRNA-296-5p, while knocking down circPSM3 promoted the proliferation and differentiation of OA chondrocytes. CircPSM3 may be a potential therapeutic target for OA treatment. Zhang et al (56) indicated that circRNA-CDR1as was markedly upregulated in OA chondrocytes compared with control chondrocytes and negatively correlated with miR-641. Furthermore, circRNA-CDR1as promoted cartilage ECM metabolism and inflammation by inhibiting the expression of miR-641 (56). Zhu et al (57) demonstrated that circGCN1L1 and TNF- $\alpha$ are upregulated in OA, while miR-330-3p is downregulated compared with healthy cartilage tissues. A further study indicated that circGCN1L1 promoted the expression of TNF- $\alpha$ by inhibiting the expression of miR-330-3p, while overexpression of TNF- $\alpha$ promoted cell inflammation and reduced the anabolism of ECM (57) (Table I).

These studies demonstrated that circRNAs serve an important role in the development of OA. CircRNAs act on different signaling molecules to promote chondrocyte apoptosis, ECM degradation and inflammation, and accelerate the process of OA. Inhibiting the expression of these signaling molecules may provide a novel direction for the treatment of OA.

\section{Inhibition of $\mathrm{OA}$ pathogenesis}

Although numerous circRNAs are known to promote the pathogenesis of OA, studies have also suggested that circRNAs such as ciRS-7, circSERPINE2 and hsa_circ_0045714 are able to inhibit the pathogenesis of OA.

Zhou et al (58) found that the expression of ciRS-7 in blood samples of patients with OA was decreased, while the expression of miR-7 was increased compared with blood from healthy controls. After IL-1 $\beta$ treatment of chondrocytes, cell proliferation was inhibited, and the release of inflammatory factors was promoted (58). The expression of ciRS-7 was downregulated in chondrocytes treated with IL-1 $\beta$, while the expression of miR-7 was upregulated. Knockdown of ciRS-7 and upregulation of miR-7 promoted inflammation and apoptosis of OA chondrocytes, which indicated that ciRS-7 exhibits a protective effect, while miR-7 promotes the development of OA (58). It was suggested that ciRS-7, a ceRNA, inhibits the expression of miR-7 and reduces apoptosis and inflammatory response of OA chondrocytes (58). This may provide novel insights for the treatment of OA.

As a member of the serine protease inhibitor superfamily, SERPINE2 is able to modify the activity of MMPs and maintain ECM homeostasis (59-61). Exons 2-4 of the SERPINE2 gene may produce circSERPINE2 through reverse splicing, which mainly includes the protein-coding sequence of SERPINE2 mRNA (59-61). Shen et al (62) reported that circSERPINE2 was low in the diseased cartilage tissue of patients with OA, while miR-1271 was highly expressed. miR-1271 was reported to be negatively correlated with the expression of SOX9, aggrecan and COL2A1 (62). The binding site of miR-1271 within ETS-related gene (ERG) is highly conserved, and miR-1271 is able to inhibit the expression of ERG (62). ERG serves an important role in inducing the permanent differentiation of chondrocytes into articular cells (63). Therefore, lack of ERG expression may increase the susceptibility of joints to damage. Inhibition of miR-1271 induces ERG overexpression and exhibited a protective role in OA (62). The results suggested that circSERPINE2 acts as a miR-1271 sponge that is able to inhibit chondrocyte apoptosis, promote ECM synthesis and prevent ECM degradation, thereby delaying the OA process (62). The circSERPINE2-miR-1271-ERG axis provides a novel direction for OA therapy. 
Table II. Roles of circRNAs in inhibiting the pathogenesis of osteoarthritis.

\begin{tabular}{|c|c|c|}
\hline CircRNA & Target & Biological function \\
\hline ciRS-7 & miR-7 & Inhibition of apoptosis and inflammation \\
\hline circSERPINE2 & $\operatorname{miR}-1271$ & $\begin{array}{l}\text { Inhibition of apoptosis, } \\
\text { promotion of ECM synthesis }\end{array}$ \\
\hline hsa_circ_0045714 & $\operatorname{miR}-193 b$ & Promotion of cell proliferation \\
\hline
\end{tabular}

Refs.

ha_circ_0045714

Promotion of cell proliferation

ECM, extracellular matrix; miR, microRNA; circ/circRNA, circular RNA.

Liu et al (42) indicated that hsa_circ_0045714 may have miR-193b binding sites, therefore, hsa_circ_0045714 may serve a role in OA by regulating miR-193b. Insulin-like growth factor (IGF)1 receptor (IGF1R), a member of the IGF family, is able to activate the PI3K and MAPK signaling pathways after binding to IGF1 and IGF2, and regulate cell proliferation, differentiation and apoptosis through autophosphorylation (64). It has been demonstrated that the division and proliferation of chondrocytes and the synthesis of Col II and proteoglycan are inseparable from IGF1R (65), which indicates that IGF1R serves a key role in chondrocyte differentiation. A dual-luciferase reporter gene assay confirmed that IGF1R is the key target gene of $\mathrm{miR}-193 \mathrm{~b}$. It has been indicated that overexpression of hsa circ_0045714 is able to inhibit the transcriptional activity of miR-193b, thereby upregulating the expression of IGF1R. IGF1R has been indicated to exhibit a similar function to hsa_circ_0045714 $(65,66)$. IGF1R promotes the expression of Col II and proteoglycans and upregulated the proliferation of chondrocytes $(65,66)$. miR-193b exhibits the opposite effect to IGF1R, as miR-193b inhibited the expression of Col II and proteoglycan, downregulated the proliferation of chondrocytes and promoted apoptosis (66). These results have suggested that hsa_circ_0045714, a ceRNA of miR-193b, inhibits the expression of miR-193b and promotes the expression of the miR193b target gene IGF1R, thereby promoting the synthesis of ECM and the proliferation of chondrocytes and inhibiting the apoptosis of chondrocytes. These results provide a novel direction for molecular targeted therapies of OA (Table II).

These studies suggest that circRNAs play an important role in the development of OA. CircRNAs act on different signaling molecules to promote the proliferation of chondrocytes, inhibit ECM degradation, reduce inflammation and delay the process of OA. Promoting the expression of these signaling molecules may provide a novel direction for delaying the progression of OA.

\section{Conclusions and perspectives}

OA is one of the most common degenerative joint diseases, with pain and activity limitation being the major symptoms. It seriously affects the quality of life of patients and places a heavy economic burden on families and society (7). Despite extensive research on OA, its pathogenesis remains to be fully elucidated and therefore, OA cannot yet be completely cured. The roles of circRNAs as regulatory molecules in the pathogenesis of OA have attracted increasing attention from scientists. These differentially expressed circRNAs provide a novel direction for the diagnosis and treatment of OA.

In addition, various signaling pathways are involved in the pathological process of OA, including PI3K/Akt and MAPK/ERK signaling pathways (64). CircRNAs exhibit a wide range of biological roles and most of their targets are essential molecules in cell signaling. Identification of novel circRNA-related signaling molecules will aid in gaining a deeper understanding of the role of circRNAs in OA, in addition to providing a theoretical basis for the targeted therapy of circRNAs.

Collectively, the present review provided a comprehensive resource that explains the important role of circRNAs in the pathogenesis of OA and reveals the interaction among various circRNAs and miRNAs in OA. Taken together, circRNAs serve a potential role in cell signaling and the pathogenesis of OA. Although experiments have demonstrated the prospective value of circRNAs in the treatment of OA, further research is required, focusing on their potential widespread use as biomarkers for OA diagnosis and on developing novel therapeutic targets for OA. The goal of this research will be to use these targets to develop novel drugs, delay the progression of $\mathrm{OA}$ and improve the quality of life of patients.

\section{Acknowledgements}

Not applicable.

\section{Funding}

No funding was received.

\section{Availability of data and materials}

Not applicable.

\section{Authors' contributions}

JW and YZ drafted and revised the manuscript. BY, CW, YG and XJ contributed to manuscript conception. Data authentication is not applicable. All authors have read and approved the final manuscript. 


\section{Ethics approval and consent to participate}

Not applicable.

\section{Patient consent for publication}

Not applicable.

\section{Competing interests}

The authors declare that they have no competing interests.

\section{References}

1. Taruc-Uy RL and Lynch SA: Diagnosis and treatment of osteoarthritis. Prim Care 40: 821-836, 2013.

2. Felson DT, Naimark A, Anderson J, Kazis L, Castelli W and Meenan RF: The prevalence of knee osteoarthritis in the elderly. The framingham osteoarthritis study. Arthritis Rheum 30: 914-918, 1987.

3. Oliveria SA, Felson DT, Reed JI, Cirillo PA and Walker AM Incidence of symptomatic hand, hip, and knee osteoarthritis among patients in a health maintenance organization. Arthritis Rheum 38: 1134-1141, 1995.

4. Prieto-Alhambra D, Judge A, Javaid MK, Cooper C, Diez-Perez A and Arden NK: Incidence and risk factors for clinically diagnosed knee, hip and hand osteoarthritis: Influences of age, gender and osteoarthritis affecting other joints. Ann Rheum Dis 73 1659-1664, 2014

5. Murphy L, Schwartz TA, Helmick CG, Renner JB, Tudor G, Koch G, Dragomir A, Kalsbeek WD, Luta G and Jordan JM Lifetime risk of symptomatic knee osteoarthritis. Arthritis Rheum 59: 1207-1213, 2008.

6. Zhang W: Risk factors of knee osteoarthritis-excellent evidence but little has been done. Osteoarthritis Cartilage 18: 1-2, 2010.

7. Glyn-Jones S, Palmer AJ, Agricola R, Price AJ, Vincent TL, Weinans H and Carr AJ: Osteoarthritis. Lancet 386: 376-387, 2015.

8. Lasda E and Parker R: Circular RNAs: Diversity of form and function. RNA 20: 1829-1842, 2014.

9. Yu CX and Sun S: An emerging role for circular RNAs in osteoarthritis. Yonsei Med J 59: 349-355, 2018.

10. Sanger HL, Klotz G, Riesner D, Gross HJ and Kleinschmidt AK : Viroids are single-stranded covalently closed circular RNA molecules existing as highly base-paired rod-like structures. Proc Natl Acad Sci USA 73: 3852-3856, 1976.

11. Hsu MT and Coca-Prados M: Electron microscopic evidence for the circular form of RNA in the cytoplasm of eukaryotic cells Nature 280: 339-340, 1979.

12. Kos A, Dijkema R, Arnberg AC, van der Meide PH and Schellekens H: The hepatitis delta (delta) virus possesses a circular RNA. Nature 323: 558-560, 1986.

13. Salzman J, Gawad C, Wang PL, Lacayo N and Brown PO: Circular RNAs are the predominant transcript isoform from hundreds of human genes in diverse cell types. PLoS One 7 e30733, 2012.

14. Jeck WR, Sorrentino JA, Wang K, Slevin MK, Burd CE, Liu J, Marzluff WF and Sharpless NE: Circular RNAs are abundant, conserved, and associated with ALU repeats. RNA 19: 141-157, 2013.

15. Hou LD and Zhang J: Circular RNAs: An emerging type of RNA in cancer. Int J Immunopathol Pharmacol 30: 1-6, 2017.

16. Chen LL and Yang L: Regulation of circRNA biogenesis. RNA Biol 12: 381-388, 2015.

17. Vicens Q and Westhof E: Biogenesis of circular RNAs. Cell 159: 13-14, 2014.

18. Wang $\mathrm{Y}$ and Wang Z: Efficient backsplicing produces translatable circular mRNAs. RNA 21: 172-179, 2015.

19. Chen I, Chen CY and Chuang TJ: Biogenesis, identification, and function of exonic circular RNAs. Wiley Interdiscip Rev RNA 6 : $563-579,2015$

20. Li Z, Huang C, Bao C, Chen L, Lin M, Wang X, Zhong G, Yu B, $\mathrm{Hu}$ W, Dai L, et al: Exon-intron circular RNAs regulate transcription in the nucleus. Nat Struct Mol Biol 22: 256-264, 2015.

21. Zhang Y, Zhang XO, Chen T, Xiang JF, Yin QF, Xing YH, Zhu S, Yang L and Chen LL: Circular intronic long noncoding RNAs. Mol Cell 51: 792-806, 2013.
22. Conn SJ, Pillman KA, Toubia J, Conn VM, Salmanidis M, Phillips CA, Roslan S, Schreiber AW, Gregory PA and Goodall GJ: The RNA binding protein quaking regulates formation of circRNAs. Cell 160: 1125-1134, 2015.

23. Ashwal-Fluss R, Meyer M, Pamudurti NR, Ivanov A, Bartok O, Hanan M, Evantal N, Memczak S, Rajewsky N and Kadener S: circRNA biogenesis competes with pre-mRNA splicing. Mol Cell 56: 55-66, 2014

24. Memczak S, Jens M, Elefsinioti A, Torti F, Krueger J, Rybak A, Maier L, Mackowiak SD, Gregersen LH, Munschauer M, et al: Circular RNAs are a large class of animal RNAs with regulatory potency. Nature 495: 333-338, 2013.

25. Hansen TB, Jensen TI, Clausen BH, Bramsen JB, Finsen B, Damgaard CK and Kjems J: Natural RNA circles function as efficient microRNA sponges. Nature 495: 384-388, 2013.

26. You X, Vlatkovic I, Babic A, Will T, Epstein I, Tushev G, Akbalik G, Wang M, Glock C, Quedenau C, et al: Neural circular RNAs are derived from synaptic genes and regulated by development and plasticity. Nat Neurosci 18: 603-610, 2015.

27. Huang S, Yang B, Chen BJ, Bliim N, Ueberham U, Arendt T and Janitz M: The emerging role of circular RNAs in transcriptome regulation. Genomics 109: 401-407, 2017.

28. Chen X, Han P, Zhou T, Guo X, Song X and Li Y: circRNADb: A comprehensive database for human circular RNAs with protein-coding annotations. Sci Rep 6: 34985, 2016.

29. Granados-Riveron JT and Aquino-Jarquin G: The complexity of the translation ability of circRNAs. Biochim Biophys Acta 1859: 1245-1251, 2016

30. Ebert MS and Sharp PA: MicroRNA sponges: Progress and possibilities. RNA 16: 2043-2050, 2010.

31. Abdelmohsen K, Kuwano Y, Kim HH and Gorospe M: Posttranscriptional gene regulation by RNA-binding proteins during oxidative stress: Implications for cellular senescence. Biol Chem 389: 243-255, 2008.

32. Yin QF, Yang L, Zhang Y, Xiang JF, Wu YW, Carmichael GG and Chen LL: Long noncoding RNAs with snoRNA ends. Mol Cell 48: 219-230, 2012.

33. Qu S, Yang X, Li X, Wang J, Gao Y, Shang R, Sun W, Dou K and Li H: Circular RNA: A new star of noncoding RNAs. Cancer Lett 365: 141-148, 2015.

34. Chen CY and Sarnow P: Initiation of protein synthesis by the eukaryotic translational apparatus on circular RNAs. Science 268: 415-417, 1995

35. Yang Y, Fan X, Mao M, Song X, Wu P, Zhang Y, Jin Y, Yang Y, Chen LL, Wang Y, et al: Extensive translation of circular RNAs driven by $\mathrm{N}^{6}$-methyladenosine. Cell Res 27: 626-641, 2017.

36. Pamudurti NR, Bartok O, Jens M, Ashwal-Fluss $R$, Stottmeister C, Ruhe L, Hanan M, Wyler E, Perez-Hernandez D, Ramberger E, et al: Translation of circRNAs. Mol Cell 66: 9-21, 2017.

37. Greene J, Baird AM, Brady L, Lim M, Gray SG, McDermott R and Finn SP: Circular RNAs: Biogenesis, function and role in human diseases. Front Mol Biosci 4: 38, 2017.

38. Hsiao KY, Sun HS and Tsai SJ: Circular RNA-New member of noncoding RNA with novel functions. Exp Biol Med (Maywood) 242: 1136-1141, 2017.

39. Li H, Yang HH, Sun ZG, Tang HB and Min JK: Wholetranscriptome sequencing of knee joint cartilage from osteoarthritis patients. Bone Joint Res 8: 288-301, 2019.

40. Xiang S, Li Z, Bian Y and Weng X: RNA sequencing reveals the circular RNA expression profiles of osteoarthritic synovium. J Cell Biochem 120: 18031-18040, 2019.

41. Xiao K, Xia Z, Feng B, Bian Y, Fan Y, Li Z, Wu Z, Qiu G and Weng X: Circular RNA expression profile of knee condyle in osteoarthritis by illumina HiSeq platform. J Cell Biochem 120 17500-17511, 2019.

42. Liu Q, Zhang X, Hu X, Dai L, Fu X, Zhang J and Ao Y: Circular RNA related to the chondrocyte ECM regulates MMP13 expression by functioning as a MiR-136 'Sponge' in human cartilage degradation. Sci Rep 6: 22572, 2016.

43. Rahmati M, Nalesso G, Mobasheri A and Mozafari M: Aging and osteoarthritis: Central role of the extracellular matrix. Ageing Res Rev 40: 20-30, 2017.

44. Loeser RF, Goldring SR, Scanzello CR and Goldring MB: Osteoarthritis: A disease of the joint as an organ. Arthritis Rheum 64: 1697-1707, 2012.

45. Marchev AS, Dimitrova PA, Burns AJ, Kostov RV, Dinkova-Kostova AT and Georgiev MI: Oxidative stress and chronic inflammation in osteoarthritis: Can NRF2 counteract these partners in crime? Ann NY Acad Sci 1401: 114-135, 2017. 
46. Sun MM, Beier F and Pest MA: Recent developments in emerging therapeutic targets of osteoarthritis. Curr Opin Rheumatol 29: 96-102, 2017

47. Laiguillon MC, Houard X, Bougault C, Gosset M, Nourissat G, Sautet A, Jacques C, Berenbaum F and Sellam J: Expression and function of visfatin (Nampt), an adipokine-enzyme involved in inflammatory pathways of osteoarthritis. Arthritis Res Ther 16: R38, 2014.

48. Salzman J, Chen RE, Olsen MN, Wang PL and Brown PO: Cell-type specific features of circular RNA expression. PLoS Genet 9: e1003777, 2013.

49. Liu Q, Zhang X, Hu X, Yuan L, Cheng J, Jiang Y and Ao Y: Emerging roles of circRNA related to the mechanical stress in human cartilage degradation of osteoarthritis. Mol Ther Nucleic Acids 7: 223-230, 2017.

50. Wu Y, Zhang Y, Zhang Y and Wang JJ: CircRNA hsa_ circ_0005105 upregulates NAMPT expression and promotes chondrocyte extracellular matrix degradation by sponging miR-26a. Cell Biol Int 41: 1283-1289, 2017.

51. Zhou ZB, Du D, Huang GX, Chen A and Zhu L: Circular RNA Atp9b, a competing endogenous RNA, regulates the progression of osteoarthritis by targeting miR-138-5p. Gene 646: 203-209, 2018.

52. Zhou ZB, Huang GX, Fu Q, Han B, Lu JJ, Chen AM and Zhu L: circRNA.33186 contributes to the pathogenesis of osteoarthritis by sponging miR-127-5p. Mol Ther 27: 531-541, 2019.

53. Chen G, Liu T, Yu B, Wang B and Peng Q: CircRNA-UBE2G1 regulates LPS-induced osteoarthritis through miR-373/HIF-1a axis. Cell Cycle 19: 1696-1705, 2020.

54. Zhu H, Zhu S, Shang X, Meng X, Jing S, Yu L and Deng Y: Exhausting circ_0136474 and restoring miR-766-3p attenuate chondrocyte oxidative injury in IL- $1 \beta$-induced osteoarthritis progression through regulating DNMT3A. Front Genet 12: 648709, 2021.

55. Ni JL, Dang XQ and Shi ZB: CircPSM3 inhibits the proliferation and differentiation of OA chondrocytes by targeting miRNA-296-5p. Eur Rev Med Pharmacol Sci 24: 3467-3475, 2020.

56. Zhang W, Zhang C, Hu C, Luo C, Zhong B and Yu X: Circular RNA-CDR1as acts as the sponge of microRNA-641 to promote osteoarthritis progression. J Inflamm (Lond) 17: 8, 2020.

57. Zhu $\mathrm{H}, \mathrm{Hu}$ Y, Wang C, Zhang $\mathrm{X}$ and He D: CircGCN1L1 promotes synoviocyte proliferation and chondrocyte apoptosis by targeting miR-330-3p and TNF- $\alpha$ in TMJ osteoarthritis. Cell Death Dis 11: 284, 2020.
58. Zhou X, Jiang L, Fan G, Yang H, Wu L, Huang Y, Xu N and Li J: Role of the ciRS-7/miR-7 axis in the regulation of proliferation, apoptosis and inflammation of chondrocytes induced by IL-1 $\beta$. Int Immunopharmacol 71: 233-240, 2019.

59. Boulaftali Y, François D, Venisse L, Jandrot-Perrus M, Arocas V and Bouton MC: Endothelial protease nexin-1 is a novel regulator of a disintegrin and metalloproteinase 17 maturation and endothelial protein $\mathrm{C}$ receptor shedding via furin inhibition. Arterioscler Thromb Vasc Biol 33: 1647-1654, 2013.

60. Pagliara V, Adornetto A, Mammì M, Masullo M, Sarnataro D, Pietropaolo C and Arcone R: Protease Nexin-1 affects the migration and invasion of C6 glioma cells through the regulation of urokinase plasminogen activator and matrix metalloproteinase-9/2. Biochim Biophys Acta 1843: 2631-2644, 2014.

61. Rao JS, Kahler CB, Baker JB and Festoff BW: Protease nexin I, a serpin, inhibits plasminogen-dependent degradation of muscle extracellular matrix. Muscle Nerve 12: 640-646, 1989.

62. Shen S, Wu Y, Chen J, Xie Z, Huang K, Wang G, Yang Y, Ni W, Chen Z, Shi P, et al: CircSERPINE2 protects against osteoarthritis by targeting miR-1271 and ETS-related gene. Ann Rheum Dis 78: 826-836, 2019.

63. Jones SW, Watkins G, Le Good N, Roberts S, Murphy CL, Brockbank SM, Needham MR, Read SJ and Newham P: The identification of differentially expressed microRNA in osteoarthritic tissue that modulate the production of TNF-alpha and MMP13. Osteoarthritis Cartilage 17: 464-472, 2009.

64. Zhang Y, Moerkens M, Ramaiahgari S, de Bont H, Price L, Meerman J and van de Water B: Elevated insulin-like growth factor 1 receptor signaling induces antiestrogen resistance through the MAPK/ERK and PI3K/Akt signaling routes. Breast Cancer Res 13: R52, 2011.

65. Zhang M, Zhou Q, Liang QQ, Li CG, Holz JD, Tang D, Sheu TJ, Li TF, Shi Q and Wang YJ: IGF-1 regulation of type II collagen and MMP-13 expression in rat endplate chondrocytes via distinct signaling pathways. Osteoarthritis Cartilage 17: 100-106, 2009.

66. Li BF, Zhang Y, Xiao J, Wang F, Li M, Guo XZ, Xie HB, Xia H and Chen B: Hsa_circ_0045714 regulates chondrocyte proliferation, apoptosis and extracellular matrix synthesis by promoting the expression of miR-193b target gene IGF1R. Hum Cell 30: 311-318, 2017.

c) (i) $\Theta$ This work is licensed under a Creative Commons International (CC BY-NC-ND 4.0) License. 